\title{
High individual heterogeneity of neutralizing activities against the original 4 strain and 9 different variants of SARS-CoV-2
}

Rita Jaafar

Aix-Marseille University

Celine Boschi

Aix-Marseille University

Sarah Aherfi

Aix-Marseille University

Audrey Bancod

Aix-Marseille University

Marion Le Bideau

AP-HM

Sophie Edouard

Aix-Marseille University

Philippe Colson

Institut Hospitalo-Universitaire Mediterranee Infection, Aix-Marseille University https://orcid.org/0000-

0001-6285-0308

Henri Chahinian

Aix-Marseille University

Didier Raoult

Aix-Marseille University

Nouara Yahi

Aix-Marseille University

Jacques Fantini

Aix-Marseille University

Bernard LA SCOLA ( $\sim$ bernard.la-scola@univ-amu.fr)

Aix-Marseille University

\section{Article}

Keywords: SARS CoV2, variants, antibodies, COVID19 vaccine, heterogeneity

Posted Date: September 2nd, 2021 
DOl: https://doi.org/10.21203/rs.3.rs-783298/v2

License: (c) (1) This work is licensed under a Creative Commons Attribution 4.0 International License. Read Full License 
2 Main manuscript for:

3 High individual heterogeneity of neutralizing activities against the original

4 strain and 9 different variants of SARS-CoV-2

5 Rita Jaafar ${ }^{1,2,3}$, Celine Boschi ${ }^{1,2,3,4}$, Sarah Aherfi ${ }^{1,2,3,4}$, Audrey Bancod ${ }^{1,2,4}$, Marion Le Bideau ${ }^{1,4}$, Sophie

6 Edouard $^{1,2,3,4}$, Philippe Colson ${ }^{1,2,3,4}$, Henri Chahinian ${ }^{3,5}$, Didier Raoult ${ }^{1,2,3,4}$, Nouara Yahi ${ }^{3,5}$, Jacques

7 Fantini ${ }^{3,5}$, Bernard La Scola ${ }^{1,2,3,4 *}$

8 Affiliations :

$9 \quad{ }^{1}$ IHU Méditerranée Infection, Marseille, France.

$10{ }^{2}$ MEPHI, Institut de Recherche pour le Développement (IRD)

$11{ }^{3}$ Aix-Marseille Université, Marseille, France.

$12{ }^{4}$ Assistance Publique - Hôpitaux de Marseille (AP-HM), Marseille, France.

$13 \quad{ }^{5}$ INSERM UMR_S 1072, Marseille, France.

14 Author contributions: BL, PC, NY, JF, DR conceived and supervised the project. RJ, CB, AB, ML 15 performed technical experiments. RJ, BL, JF, NY, HC, SA \& PC interpreted and analysed the results. RJ $16 \&$ BL wrote the manuscript. CB \& SE collected human plasma samples. All authors amended and agreed 17 to the publication of this version of the manuscript.

18 Competing interest statement: The authors declare no competing interest.

19 Classifications: Biological sciences (Major), Microbiology (Minor)

20 Keywords: SARS CoV2, variants, antibodies, COVID19 vaccine, heterogeneity

21 * Corresponding author: Bernard La Scola: bernard.la-scola@univ-amu.fr (BL)

22 IHU - Méditerranée Infection, 19-21 boulevard Jean Moulin, 13005 Marseille, France. Tel.: +33

23413732 401, Fax: +33413732402.

\section{This PDF Includes:}

25 Main text

26 Figure 1 to 3

27 Table 1 


\section{Abstract}

29 Background. Since the beginning of the COVID-19 pandemic, several SARS-CoV-2 variants have sequentially emerged. In France, most cases were due to spike D641G-harbouring viruses that descended initially from the Wuhan strain, then by variant of B.1.160 lineage we called Marseille-4 since the summer of 2020, which was followed by the alpha (UK) and beta (South African) variants in early 2021, then delta (Indian) now.

Methods and Findings. We determined the neutralizing antibody (nAb) titres in sera from convalescent individuals previously infected by these 4 major local variants and from vaccine recipients to the original Wuhan strain and 9 variants, including two recent circulating delta (Indian) isolates. The results show high inter-individual heterogeneity in nAbs, especially according to the variant tested. Unexpectedly, the major variations among nAbs are based on the genotype responsible for the infection. Patients previously infected with the beta and B.1.160 variants had the lowest nAb titres. We show that this heterogeneity is well explained by spike protein mutants modelling using in silico approaches. The highest titres were observed in patients vaccinated with the Pfizer/BioNTech COVID-19 vaccine, even against the delta variant.

Conclusions. Immunity acquired naturally after infection is highly dependent on the infecting variant and unexpectedly mRNA-based vaccine efficacy is shown to be often better than natural immunity in eliciting neutralizing antibodies.

\section{Significance statement}

47 With the ongoing rapid evolution of SARS CoV 2, understanding the neutralizing activity against current and potential future variants is now considered crucial to protection provided by natural infection and vaccine to prevent reinfections. In this study, we analysed the reactivity by seroneutralization test towards 10 different SARS-CoV-2 strains in sera from patients with previous natural infection and individuals immunized by two injections of the SARS CoV 2 vaccine. As a result, we demonstrated high inter-individual heterogeneity in nAbs. Our data showed that the nAbs acquired naturally after infection were highly dependent on the variant causing the infection. Our data indicate that the mRNA-based vaccine efficacy is often better than natural immunity in eliciting neutralizing antibodies. 


\section{Introduction}

SARS-CoV-2 is the seventh member of the Coronaviridae family that infects humans and causes COVID-19. As of June 1, 2021, more than 165 million infections with approximately 3.4 million deaths have been recorded [1]. In France, despite declining numbers of COVID-19 patients in intensive care units, controlling and preventing the spread of the virus remains crucial [2]. Across the world, vaccines have been developed and commercialized, and the implementation of vaccination strategies and policies has become a priority.

The practice of immunization dates back hundreds of years. In 1798, the first smallpox vaccine was developed, and since then, multiple vaccines have been developed and are available. However, during the last three decades, molecular genetics as a focus in immunology, microbiology and genomics have been applied to the field of vaccinology [3]. This has led to the development of new vaccine types and delivery systems, such as DNA, RNA, and viral vectors, as well as an inactivated or even live attenuated forms of viral or bacterial pathogens [4]. As of 2 May 2021, a total of 187,490,581 doses of COVID-19 vaccines have been provided to countries in the European Union and the European Economic Area (EU/EEA) [5]. Following several announcements by several manufacturers of the COVID-19 vaccine that the clinical trials have shown the efficacy of the COVID-19 vaccine for preventing SARS-CoV-2 infection, authorities have now required a comprehensive post-efficacy strategy for the next steps to ensure vaccination of the global population. Of these vaccines, $65.6 \%$ of all doses distributed to EU/EEA countries were with the Pfizer/BioNTech COVID-19 vaccine (BNT162b2), followed by AZD1222, previously named the COVID-19 Vaccine by AstraZeneca (23.8\%), the COVID19 Vaccine by Moderna (8.9\%) and the COVID-19 Vaccine by Janssen (1.1\%) [5]. The remaining questions are whether the antibodies generated after vaccination and after natural infection confer long-lasting immunity and whether rapidly evolving mutants, especially those with spike protein mutations, modify the vaccines' effectiveness.

Four new variants that have rapidly become dominant in the mentioned countries have 
B.1.351 variant that was first detected in South Africa, the B.1.1.28.1 (P.1) variant that first spread in Brazil, and the B.1.617.2 variant that was first identified in India. These variants that emerged between late 2020 and the beginning of 2021 are now classified by the CDC as "variants of concerns" (VOCs) due to their transmissibility, mortality and immunogenicity characteristics. However, other variants, such as the Marseille-4/B.1.160 variant, were responsible for a large number of cases and associated deaths such as in France [6]. Although policies to prevent the spread of SARS-CoV-2 variants are implemented, the high mutation rate and rapid emergence of variants of this RNA virus highlight the importance of vigilance with regard to the genomic surveillance for the early identification of future variants. Eliciting broadly neutralizing activity against current and potential future variants is now considered a must to evaluate vaccine efficiency and to prevent reinfections. analysis of the spike proteins expressed by the different SARS-CoV-2 strains studied.

100

101

\section{Results}

102

\section{Convalescent plasma}

The IgG titres provided by the CLIA test are shown in Figure 1, Figure 2, and Supporting Table S4. According to the manufacturer, output results were considered positive for the presence of anti-SARS-CoV-2-IgG antibodies for values $>15 \mathrm{AU} / \mathrm{mL}$, negative for values $<12 \mathrm{AU} / \mathrm{mL}$, and as borderline for values between 12-15 AU/mL. Among convalescent COVID19 patients tested by CLIA, 34/40 (85\%) sera were IgG positive, 2/40 (5\%) (SA-1, SA-6) were considered borderline, and 4/40 (10\%) were negative (Supporting Table S1). By arbitrarily classifying the patients with high titres of antibodies (IgG titres >100), the prevalence of the high titres decreased among patients infected by the original/B genotype (8/9, 89\%), the UK/B.1.1.7 genotype $(3 / 10,30 \%)$, the Marseille-4/B.1.160 genotype $(1 / 9,11 \%)$ and the South 
African/B.1.351 genotype (0/12, 0\%). Moreover, a significant proportion $(3 / 12,25 \%)$ of the patients infected with the South African/B.1.351 variant were negative for IgG by CLIA, and these samples represented $75 \%$ of all samples that tested negative.

For MNT, the observed titres were low, ranging from no seroneutralization $(<1 / 5)$ to 1/160 (Figure 1). The same was found for CLIA in terms of the reactivity of the different groups of convalescent sera. By arbitrarily classifying the patients with high $\operatorname{IgG}$ antibody titres $\geq 1 / 10$ against the strain that the patients were infected with, the decreasing order of the prevalence of titres were: the original/B genotype $(9 / 11,82 \%)$, UK/B.1.1.7 genotype $(8 / 10,80 \%)$, Marseille4/B.1.160 genotype $(2 / 9,22 \%)$ and South African/B.1.351 genotype (3/12, 25\%). The presence of neutralizing antibodies in each group of patients against the variants, excluding those variants responsible for the patients' infection, varied according to the variant tested. Without taking into account the sera of patients convalescing for the South African/B.1.351 variant that react nearly only to South African/B.1.351 and Brazilian/B.1.1.28.1 variants, at a 1/5 MSN titre, 14/30 (47\%) of the samples reacted against the Original/B genotype, 10/19 (53\%) reacted against the Marseille-4/B.1.160 genotype, 13/20 (65\%) reacted against the UK/B.1.1.7 genotype, 20/30 (66\%) reacted against the Marseille-501/A.27 variant, 18/30 (60\%) reacted against the Brazilian/B.1.28.1 variant, 15/60 (50\%) reacted against the Marseille-484K.V1/R.1 variant, 24/30 (80\%) reacted against the Belgian/B.1.214 variant and 12/60 (50\%) reacted against the Indian B.6K.V1 variant. The South African/B.1.351 variant was the least recognized variant after excluding the variants that caused the infections, as only 8/30 (27\%) had detectable seroneutralising antibodies against this variant.

\section{Vaccinated patients' sera}

Regarding the vaccinated participants and their CLIA serology test results, both participants who received the 2 AZD122 injections were IgG positive, and the majority (11/12) of the participants were vaccinated with 2 doses of the Pfizer/BioNTech vaccine (Figure 1, Figure 2 and Supporting table S5). However, one patient (V-Pfizer-10) who showed no detectable reaction by the seroneutralization tests, even against the original Original/B strain despite the patient previously receiving 2 doses of the Pfizer/BioNTech vaccine, was also negative for $\mathrm{IgG}$ in the CLIA. This patient was not immunocompromised but was an elderly 
141 patient (88 years old). The other patient with a low antibody titre (V-Pfizer 4) had a previous 142 splenectomy.

The neutralizing profiles of most patients who had the Pfizer/BioNTech vaccine also showed neutralization gaps in the South African/B.1.351 variant (Figure 1 and Figure 2). Otherwise, these sera appeared to inhibit the in vitro CPE for 9 out of the 10 SARS-CoV-2 strains until the sera dilutions were 1:40 and 1:80. Both persons who received two shots of the AZD122 vaccine displayed limited to completely absent neutralization on all the tested SARSCoV-2 isolates. One Astra-2 serum showed a stronger reaction with the Belgian/B.1.214 variant isolate. The results of individuals who received the Pfizer/BioNTech vaccine also showed heterogeneity in the neutralization profiles, as some had much weaker antibody titres than those vaccinated and had very high $\operatorname{IgG}$ titres (>400 AU/mL). Of interest for the current period, 8/11 patients vaccinated by the Pfizer/BioNTech vaccine had MSN titres $\geq 1 / 10$ against both of the Indian/B.1.617.2 variant strains tested.

\section{Human monoclonal antibody LY-CoV555}

We assayed the neutralizing activity of the commercial monoclonal antibody bamlanivimab (LY-CoV555) at an initial concentration of $35 \mathrm{mg} / \mathrm{mL}$, and the results of the neutralization activity of this tested mAb are summarized in the first row in Supporting Figure S1. LY-CoV555 significantly neutralized the Original/B strain and the Marseille-4/B.1.160 variant (neutralizing titre of $0.224 \mu \mathrm{g} / \mathrm{mL}$ ) and less significantly neutralized the UK/B.1.1.7 and Belgian/B.1.214 variants (neutralizing titre of $1.12 \mu \mathrm{g} / \mathrm{mL}$ ). Additionally, it had almost no neutralizing activity on the Marseille-501/A.27 and Indian/B.1.617.2 variants (very low neutralizing titre of $3500 \mu \mathrm{g} / \mathrm{mL}$ ). Moreover, the South African/B.1.351, Brazilian/B.1.1.28.1 and Marseille-484K.V1/R.1 variants were profoundly resistant to neutralization by bamlanivimab (LY-CoV555).

\section{Molecular mechanisms of the neutralization escape of SARS-CoV-2 variants}

Most neutralizing antibodies (nAbs) against SARS-CoV-2 are directed against the RBD and the NTD of the spike protein. As references, we used the LY-CoV $555 \mathrm{nAb}$ (bamlanivimab) 
168

169

170

171

172

173

174

175

176

177

178

179

180

181

182

183

184

185

186

187

188

189

190

191

192

193

194

195

196

197

and the $4 \mathrm{~A} 8 \mathrm{nAb}$, which recognize the principal neutralization determinants of the RBD and the NTD, respectively.

The E484K substitution (Glu $\rightarrow$ Lys substitution) in the Marseille-484K.V1/R.1 variant induces a dramatic rearrangement of the RBD surface that results in a complete lack of interaction with the bamlanivimab nAb (Supporting Figure S2A and S2B). This molecular mechanism explains the dramatic decrease in the affinity $(85 \%)$ of the bamlanivimab nAb for the RBD of the Marseille-484K.V1/R.1 variant (Table 1). A similar mechanism also accounts for all variants that display the E484K mutation, including the South African/B.1.351 (70\% decrease) and Brazilian/B.1.1.28.1 (67\% decrease) variants (Table 1).

The L452R substitution is present in both the Marseille-501/A.27 (Supporting Figure S2C) and the Indian/B.1.617.2 (Supporting Figure S2D) variants, yet in a distinct mutational context. In the case of the Marseille-501/A.27 variant, the L452R mutation is associated with N501Y. The loss affinity of Bamlanivimab's nAb for this variant was estimated to be $76 \%$ (Table 1). The molecular mechanism of this effect could be attributed to a reorientation of the cationic side chain of R452 (compared to L452), which takes Y449 away from the antibody heavy chain residue N31 (Supporting Figure S3A and S3B).

The case of the Indian/B.1.617.2 variant is more puzzling since, in this case, the substitution L452R is associated with T478K instead of N501Y. As shown in Supporting Figure S4A, in the Original/B strain, T478 is close to F486, a key amino acid controlling bamlanivimab recognition. Indeed, the methyl group of T478 points in the direction of the aromatic ring of F486, which allows the formation of a cluster of $\pi-\pi$ interactions with Y32 and Y92 of the light chain of the antibody. The clamp of Y32 and Y92 is particularly visible when the amino acid atoms are represented in spheres (Supporting Figure S4A, upper panel). When T478 is substituted by K478 (T478K substitution), F486 is attracted by the cationic group of K478, preventing any contact with the aromatic amino acids Y32 and Y92 of the antibody (Supporting Figure S4B). This mechanism largely contributes to the $72 \%$ loss of affinity of bamlanivimab for the RBD of the Indian/B.1.617.2 variants (Table 1).

Finally, we evaluated the impact of mutations in the NTD on antibody recognition (Table 1). Interestingly, some mutational patterns did not seem to decrease the affinity of the $4 \mathrm{~A} 8 \mathrm{nAb}$ for the NTD, and in some cases, the affinity was even slightly increased, as shown for the 
Marseille-484K, V1/R1 and UK/B.1.1.7 variants (Table 1). In other cases, a significant decrease in the antibody affinity, compatible with the neutralization escape, was calculated and ranged from $64 \%$ for the Brazilian/B.1.1.28.1 variant to $47 \%$ for the South African/B.1.351 variant (Table 1). At the opposite end of the scale, the affinity of 4A8 for the UK/B.1.1.7 variant was slightly increased (-241 kJ.mol ${ }^{-1}$ vs. $-225 \mathrm{~kJ} \mathrm{~mol}^{-1}$ for the Original/B strain). A detailed analysis of the 4A8 epitope provided a molecular explanation for such a range of effects (Figure 3). This epitope is divided into two prominent and flexible regions of the NTD, the N3 and N5 loops, which adopt a crescent-like shape recognized by the antibody (Figure 3A). Key residues involved in 4A8 binding belong either to the N3 loop (K147, K150 and W152) or to the N5 loop (R246, Y248 and L249). Inasmuch as both loops are accessible at the NTD surface, the 4A8 antibody can bind to the NTD, as shown for the Origjnal/B strain (Figure 3A) and the UK/B.1.1.7 variant (Figure 3B). In the case of the South Africa/B.1.351 variant, the only part of the epitope preserved from this dramatic reorganization of the NTD is the tip of the N3 loop harbouring K147 and K150, which may explain the residual affinity of some anti-NTD nAbs (such as those elicited by vaccination) for this variant (Figure 3C). However, this truncated epitope may lose most of its immunogenicity. Thus, patients infected by the Original/B or the UK/B.1.1.7 strains may elicit nAbs against several variants, including the South African/B.1.351, but the reverse is not true, as sera from patients infected by the South African/B.1.351 variant have poor neutralizing activities. Subtle conformational changes in the NTD affecting the relative orientations of the K147 and R246 side chains were consistent with the slightly decreased affinity of anti-NTD nAbs for the Indian 2 variant vs. the Indian 1 variant (Supporting Figure S5). Since both variants have the same RBD but display distinct mutational patterns in the NTDs, these data underscored the importance of the NTD as a key neutralizing determinant of SARS-CoV-2.

\section{Discussion}

In this study, we report a serological investigation using a CPE-based microneutralization assay of anti-SARS-CoV-2 antibodies and tested 10 different strains of this virus including the original strain that initially spread and 9 variants. Seroneutralization assays are always the gold 
standard for in vitro assays. This technique performed with replication-competent coronaviruses seems to have an epidemiological potential to detect the presence or absence of neutralizing antibodies against the newly emerging SARS-CoV-2 variants. This technique is clearly superior to pseudoviral systems that do not reflect a real viral infection since they only mimic the entry step of the virus' life cycle and have serious limitations related to the usage of unnatural core proteins[14]. We managed to obtain convalescent plasma samples from 42 patients at least 3 weeks after a documented SARS-CoV-2 infection. As illustrated in Figure 2, the overall antibody response was divided into 4 groups, each group representing a period of infection.

A remarkable neutralization pattern with high antibody levels was seen in convalescent patients recovered from the original Original/B strains (lineage B), as determined by CLIA. Afterwards, the humoural response was also detected in patients previously infected with the UK/B.1.1.7 variant between March and April 2021, and these patients had moderate levels of IgG following the infection. Additionally, antibody titres were shown to be reduced to low levels in patients previously infected with the Marseille-4/B.1.160 variant, and very low IgG levels were detected in the plasma of patients recovered from infection with the South African/B.1.351 variant.

Two groups of patient antibodies seem to significantly recognize the strain causing the infection, as seen in the case of Original/B strain patients and UK/B.1.1.7 variant patients. This was not seen in patients who had recovered from an infection with the Marseille-4/B.1.160 and South African/B.1.351 variant strains. However, some positive IgG sera in the patients infected with the B.1.351 variant contained antibodies that are able to neutralize this variant, albeit poorly (neutralization titres ranging mostly from 1:5 and 1:10). This variant is particularly interesting because it displays both single point mutations and deletions, which induce a global reorganization of the NTD. However, the tip of the N3 loop harbouring K147 and K150 was only marginally affected by this reorganization, which may explain the residual affinity of some nAbs for the NTD.

Regarding the global humoural response, our data highlight a strong variability in the antibody levels and in the neutralization profiles. The reason behind this "interindividual heterogeneity" is not yet clear. However, multiple studies have shown a sort of positive correlation between the serum neutralizing capacity and disease severity, highlighting the highly 
257 heterogeneous nature of nAb responses against the SARS-CoV-2 spike protein [15,16]. In 258 response to the question of what the immunity and protection levels are following a natural infection against the currently circulating variants, especially the VOCs, we also studied the reactivity between $\mathrm{nAbs}$ and 9 different variant isolates. Our results show that infection with the Marseille-4/B.1.160 and the South African variants did not confer humoural protection against the majority of the circulating strains. However, a relative but significant immunity was observed for those patients who recovered from the original strain that circulated between March and June 2020 as well as the patients who had the UK/B.1.1.7 variant in reactions against all of the strains except for the South African strain. Our data suggest that this variant strain (B.1.351) is ultimately resistant to the activity of nAbs. This in vitro resistance correlates with the first reinfection case reported in France by February 2021 by the South African/B.1.351 SARS-CoV2 VOC (beta, V2), which caused a severe case of COVID-19 4 months after the first mild infection [17]. Our findings also correlate with another in vitro assay confirming that B.1.351 may escape the neutralizing antibody response elicited by prior natural infection with a half maximal inhibitory concentration (IC50) 6 to 200 times higher than that of the virus in the first wave of the pandemic [18]. A potential hypothesis may have arisen concerning intrahost evolution in some individuals with sustained viral replication where the genetic diversity from a continuous turnover of dominant viral species may have resulted from differential selective pressures $[19,20]$. As the receptor binding motif $(\mathrm{RBM})$ is considered the main functional motif that forms the interface with the human ACE2 (hACE2) receptor, multiple studies have shown that the corresponding epitope mutation centred around E484 led to various amino acid changes and strongly affected plasma antibody neutralization [21-25]. This can be seen for the Brazilian/P.1 and Marseille-484K.V1/R.1 variants harbouring the same spike key mutation (E484K), as they show a similar but potential immune escape (with low nAb recognition). The last combination of mutations that are currently spreading worldwide was previously identified as the new B.1.617.2 variant that first emerged in India in October 2020 and spread further in many countries. Due to its key spike protein mutations (L452R and T478K), the Indian variant may induce an immune evasion [26], similar to the B.1.351, P.1 and R.1 variants. As shown in Table 1, the B.1.617.2 variant strain seems to be resistant to recognition by the LY-CoV555 (bamlanivimab) monoclonal antibody that we tested in our study. These findings are similar to those recently described in a new preprint that was available on the bioRxiv preprint website at 
the end of May 2021 [27]. The preprint showed that there was a reduced sensitivity of the B.1.617.2 variant to the sera from convalescent patients and vaccinated individuals, and our results on the sera of patients previously infected with the original Original/B strain UK/B.1.1.7 and the Marseille-4/B.1.160 variants also had less reactivity towards this variant.

In our study, we finally tested the mAb named LY-CoV555, which was authorized for emergency use by the FDA. By April 16th, 2021, the FDA revoked the emergency use authorization (EUA) [28] that allowed the investigational monoclonal therapy by bamlanivimab to be used [29]. Based on new data and ongoing analyses in addition to the increase in SARSCoV-2 viral variants that are shown to be resistant to mAbs, therapy with bamlanivimab alone [30] has resulted in an increased risk for treatment failure. Although our data confirm these conclusions, as we see in Table 1, bamlanivimab neutralizes only the UK/B.1.1.7 variant, Marseille-4/B.1.160, the Belgian variant and the original Original/B strain. For other VOCs, no neutralizing activity of these mAbs was observed.

Our molecular modelling data are in complete agreement with this finding (Table 1). The variants that are neutralized by bamlanivimab are well recognized by this antibody, with $\Delta \mathrm{G}$ values ranging from -195 to $-245 \mathrm{~kJ} \cdot \mathrm{mol}^{-1}$, which is close to the $\Delta \mathrm{G}$ of the reference Original/B strain $\mathrm{nAb}$ complex $\left(-244 \mathrm{~kJ}_{\mathrm{mol}}{ }^{-1}\right)$. In contrast, variants that resist bamlanivimab seroneutralization (Marseille-484K.V1/R.1, Marseille-501/A.27, Brazilian/B.1.1.28.1, Indian/B.1.617.2, South African/B.1.351) have very low affinity for the $\mathrm{nAb}$, with values of $\Delta \mathrm{G}$ ranging from -36 to $-80 \mathrm{~kJ} \mathrm{~mol}^{-1}$. The analysis of the NTD-nAb complex of each variant confirmed this classification, although the NTD appeared to display interesting features. In general, the loss of affinity of the RBD for nAbs was associated with a similar loss of affinity of the NTD for its own nAbs. The only exception to this rule was the Marseille-484K.V1 variant because it did not display any mutation in the NTD. In all other cases, there was a good correlation between the neutralization escape of the RBD and of the NTD. However, residual $\Delta \mathrm{G}$ values were globally higher for variant NTDs than for variant RBDs. In fact, the affinity of variant NTDs was decreased by 64\% at maximum (Brazilian/B.1.1.28.1 variant), compared to $85 \%$ in the case of the RBD (Marseille-484K. V1 variant). For the South African variant, the loss of affinity of the anti-NTD nAb was estimated to be $48 \%$ (Table 1). This finding is in good 
agreement with the seroneutralization data, which showed that $42.9 \%$ of fully vaccinated individuals could not neutralize the South African variant [31].

Structural analysis of the $4 \mathrm{~A} 8$ epitope revealed that it is partially formed by a flexible loop of the NTD (the N3 loop) that may remain accessible for the nAb even in the presence of multiple mutations and/or deletions in the NTD (Figure 3). Thus, the sensitivity of a given variant to seroneutralization may depend on the relative balance between anti-RBD and antiNTD nAbs in the patients' sera. This balance may in fact explain the discrepancy in seroneutralization studies that concluded that SARS-CoV-2 variants could be either partially or totally resistant to nAbs [24,32]. Initially, it was assumed that most anti-SARS-CoV-2 nAbs were directed against the RBD [33]. A more recent analysis [34] challenged this view and concluded that the prevalence of anti-NTD neutralizing antibodies was higher than the anti-RBD nAbs in convalescent subjects. Indeed, more than $80 \%$ of the immunological response lies outside the RBD. Combined with our modelling study, these data suggest that the heterogeneity of SARS-CoV-2 seroneutralization mostly reflects the neutralizing activity of anti-NTD antibodies. The lower the anti-NTD nAb titre, the higher the immunological escape. It is also important to note that several variants (e.g., South African variant) have a lower accessibility of the N5 loop to nAbs (Figure 3), so that any therapeutic anti-NTD monoclonal antibody directed against this loop may be of limited use [35].

Structural analysis of the SARS-CoV-2 spike protein in conjunction with nAbs and molecular modelling studies of variants can help determine which variants of interest (VOIs) may become variants of concern (VOCs). This variant status can be estimated in real time from genome sequence data by calculating the transmissibility (T-index) [13] and, for the first time, the immune escape (I-index) capabilities. This I-index takes into account the impact of mutations in the RBD and of mutations/deletions in the NTD on the free energy variation $(\Delta \mathrm{G})$ of each nAb-spike complex (Table 1). It perfectly separated the variants that escape the binding of antibodies, and by introducing an optimized I-index, it was possible to perfectly match it with the reactivity observed in the patients and thus predict the capability to escape antibodies of any upcoming SARS-CoV-2 strain.

The results from phase III clinical trials in the United Kingdom revealed that the BNT162b2 and AZD1222 vaccines were highly effective when using a two-shot protocol with a 
target interval of three and four weeks, respectively, between doses[36]. In addition to immunity following natural infection, we were interested in studying the acquired immunity following vaccination. In Europe and especially in France, vaccination and immunization will now be available for all individuals that are older than 18 years of age [37]. Two doses of the vaccines are required to achieve adequate immunization against COVID-19. Our data show that patients vaccinated with the mRNA-based vaccine have a promising neutralizing profile with variable but interestingly high nAb titres, even with most variants, as described previously [37]. As recently observed, the new Indian variants are also neutralized by serum antibodies (sera which have variable antibody titres) that are "individual-dependent", and these can be neutralized with lowered titres [27]. These data allow us to conclude that mRNA-based vaccine efficacy is even better than natural immunity in eliciting neutralizing antibodies. In our study, we could not make the same conclusion for the AZD1222 vaccine, as we could not test more than 2 sera due to the long waiting period, which exceeded our study timeframe. However, we observed that neither the serum that had high IgG titres by CLIA nor the sera that had high neutralizing profiles by seroneutralization reacted with the 10 SARS-CoV-2 strains. Despite the promising viral neutralization profiles of vaccinated individuals with the Pfizer/BioNTech vaccines in our cohort, a recent sero-epidemiological study showed that reinfection among patients previously infected by SARS-CoV-2 occurs at a lower rate $(0.23 \%)$ than infection occurrence within previously vaccinated patients $(5.1 \%)$ [38]. These findings are inconsistent with the outcomes obtained by the seroneutralization tests, but viral neutralization tests consist of in vitro approaches that may not reflect the effect of cellular immunity within the human body $[39,40]$, as this technique is based exclusively on antibody-antigen interactions. A study published in May 2020 reported that during a COVID-19 infection, the SARS-CoV-2 spike (S) protein was found to be a nondominant target of the human CD8+ T cell response and that the recognition of the SARS-CoV-2 M (Matrix) antigen was similarly strong to the $\mathrm{S}$ antigen, which is unlike other coronaviruses [41]. Additionally, significant reactivity was found for other antigens, most notably the nsp6, ORF3a, and N antigens. Subsequently, COVID-19 vaccines that target only one antigen (the spike protein) will elicit a relatively narrow cellular response when compared with natural-induced $\mathrm{T}$ cells, which can target more than one antigen in convalescent patients [41,42]. Moreover, the large amounts of SARS-CoV-2 spike protein provided by mRNA- or adenovirus-based vaccines generally give rise to high titres of anti-spike circulating IgG [38]. As 
confirmed in a previous cohort performed in our institute, the SARS-CoV-2 spike protein is not the only immunogenicity marker for SARS-CoV-2 infection, as validated by automated western immunoblotting assays [43]. In naturally infected patients, lower IgG titres are detected, probably because the immunological antibody response involves primarily mucosal IgA. Our seroneutralization data are thus consistent with this difference in the $\mathrm{nAb}$ titres between naturally infected and vaccinated individuals.

\section{Materials and methods}

\section{Serum samples and human monoclonal antibodies}

A total of 55 human serum samples were included as part of a sero-epidemiological study that is being performed in our laboratory, and the patients' sera included 42 sera obtained from convalescent patients within 3 weeks to 5 months after a documented COVID-19 infection (Supporting Table S1 and Supporting Table S2). Eleven patients were infected by spike D614G-harbouring B lineage strains that spread during the first wave of COVID-19 infections in France, 9 were infected by the Marseille-4/B.1.160 variant, 10 were infected by the UK/B.1.1.7 variant and 12 were infected by the South African/B.1.351 variant. Direct genotyping from respiratory samples was performed under previously described conditions [7]. Along with these sera, 13 sera from vaccinated individuals were also selected. Eleven of these individuals received 2 shots of the Pfizer/BioNTech COVID-19 vaccine (BNT162b2), and two received 2 shots of the AZD1222 COVID-19 vaccine. These vaccinated individuals were sampled within two to twelve weeks after their second shot. For the human monoclonal antibody, we selected LY-CoV555 to be tested along with the serum samples in the microneutralization test (MNT) and used it as our positive control for the tested antibodies in the established assays.

\section{Ethical statement}

This study was approved by the Ethics Committee of the IHU Mediterranée Infection under the number 2021-011. The serum samples were collected for diagnostic purposes and were reused for the MNT anonymously. According to French law (loi Jardé), anonymous retrospective studies do not require institutional review board approval. 


\section{Serological IgG test}

Specific anti-SARS-CoV-2 IgG antibodies were detected by the Liaison XL automated chemiluminescent immunoassay (CLIA) (Diasorin Inc., Saluggia, Italy) according to the manufacturer's recommendations. This test uses magnetic beads coated with antigens derived from subunits $\mathrm{S} 1$ and $\mathrm{S} 2$ of the viral spike protein.

\section{Cell line preparation and subculturing procedure}

Vero E6 cells (ATCC-CRL-1586) were propagated and cultured in minimal essential medium (MEM, Gibco, USA) supplemented with $2 \mathrm{mM}$ L-glutamine and $10 \%$ foetal bovine serum $(\mathrm{FBS})$ at $37^{\circ} \mathrm{C}$ in a $5 \%$ humidified incubator. Ninety-six-well plates of Vero E6 cells were prepared for the neutralization tests of SARS-CoV-2 in MEM growth medium supplemented with glutamine and $4 \%$ FBS.

\section{Viral strains}

The viruses used in our study correspond to the strains isolated at our laboratory, IHUMéditerranée Infection, as a part of routine virology work. Viral strains were isolated in cell culture from patients' clinical samples under previously described conditions and then frozen at $80^{\circ} \mathrm{C}$ for further use [8]. All strains were confirmed as SARS-CoV-2 and genotyped by whole genome next-generation sequencing as previously described [5] (Supporting Table S3). For the microneutralization test (MNT), virus production was performed by thawing the previously conserved virus suspension and reinoculating in a previously prepared 12-well Vero E6 cell plate at a density of $4 \times 10^{5}$ cells $/ \mathrm{mL}$. After $48 \mathrm{~h}$, the virus suspension was harvested and quantified by real-time reverse-transcription (RT)-PCR (qPCR) and TCID50 determination.

\section{Micro-neutralization test (MNT)}

Our study was based on a cytopathic effect (CPE)-based MNT. Each serum sample was assayed for neutralization against the 10 SARS-CoV-2 strains. Sera were heat inactivated at $56^{\circ} \mathrm{C}$ for $1 \mathrm{~h}$. Twofold serial dilutions from 1:5 to 1:640 were prepared and then mixed with each of the 10 tested viral strains that had been previously quantified by qPCR and normalized to a cycle threshold value $(\mathrm{Ct})$ of 25 through dilution of the viral stock with culture medium (MEM, 
supplemented with $4 \%$ FBS and $2 \mathrm{mM}$ glutamine). This normalization was verified by reading the TCID50 at 5 days and corresponded to $4.35 \pm 0.23 \log _{10}$ virus $/ \mathrm{mL}$ for all strains. The serum/virus mixture was incubated for $1 \mathrm{~h}$ at $37^{\circ} \mathrm{C}$ in a humidified atmosphere with $5 \% \mathrm{CO}_{2}$. After incubation, $100 \mu \mathrm{L}$ of cell culture medium was removed, and $100 \mu \mathrm{L}$ of the mixture at each dilution was added in quadruplicate to a 96-well cell plate containing a subconfluently Vero E6 cell monolayer. The plates were incubated for 5 days at $37^{\circ} \mathrm{C}$ in a humidified atmosphere with $5 \% \mathrm{CO}_{2}$. The same procedure was established for the human monoclonal antibody, except for the antibody dilution that corresponded in 1:5 serial dilutions going from a concentration of $3,500 \mu \mathrm{g} / \mathrm{mL}$ to $0.0089 \mu \mathrm{g} / \mathrm{mL}$ of LY-CoV555. After 3-5 days of incubation, the plates were inspected by an inverted optical microscope. On the $5^{\text {th }}$ day, the highest serum dilution that protected at least $50 \%$ of cells from $\mathrm{CPE}$ was taken as the neutralization titre.

\section{Statistical tests}

We performed a statistical analysis using GraphPad Prism v9.0.0 (GraphPad Software, LaJolla, California, USA) using an analysis of variance (ANOVA), followed by Tukey's multiple comparisons test. P-values $\leq 0.05$ were considered as significant.

\section{Computational methods}

The spike protein mutants were modelled using in silico approaches. As the mutations are localized in two different domains of the spike, namely, the N-terminal domain (NTD) and the receptor binding domain (RBD), separate models were generated for each domain. The atomic coordinates of the RBD bound to LY-CoV 555 neutralizing antibody (nAb) were retrieved from PDB file 7KMG [9], and the structure of the NTD bound to the $4 \mathrm{~A} 8 \mathrm{nAb}$ was retrieved from PDB file 7C2 L [10]. Minimized structures of the RBD and NTD of each variant were obtained by introducing appropriate mutations and/or deletions in the initial PDB files. Energy minimizations of the variants were performed with the Polak-Ribière conjugate gradient algorithm with the Bio-CHARMM force field in Hyperchem [11] using a maximum of $3 \times 10^{5}$ steps and a root-mean-square (RMS) gradient of $0.01 \mathrm{kcal} . \AA^{-1} \cdot \mathrm{mol}^{-1}$ as the convergence condition. The variant domain was then merged with the corresponding nAb (LY-CoV555 for the RBD, 4A8 for the NTD) using the initial coordinates of the 7KMG and 7C2 L pdb files, and the whole system was succumbed to a new series of energy minimizations. The energy of 
462 interaction of each complex was calculated with a Molegro molecular viewer as previously 463 described $[12,13]$.

\section{Acknowledgments}

465 We sincerely thank Cedric Mantelli for the technical help. We also acknowledge the contribution 466 of the technical staff of the the IHU Méditerranée Infection Laboratory.

\section{Funding sources}

468 This work was supported by the French Government under the "Investments for the Future" 469 program managed by the National Agency for Research (ANR), Méditerranée-Infection 10470 IAHU-03 and was also supported by Région Provence-Alpes-Côte d'Azur and European funding 471 Fonds Européen de Développement Régional - Plateformes de Recherche et d'Innovation 472 Mutualisées Méditerranée Infection.

473

\section{References:}

475 1. COVID-19 situation update worldwide, as of week 27, updated 15 July 2021. Available at: 476 https://www.ecdc.europa.eu/en/geographical-distribution-2019-ncov-cases. Accessed 22 477 July 2021.

478 2. Coronavirus : chiffres clés et évolution de la COVID-19 en France et dans le Monde. 479 Available at: https://www.santepubliquefrance.fr/dossiers/coronavirus-covid480 19/coronavirus-chiffres-cles-et-evolution-de-la-covid-19-en-france-et-dans-le-monde. $481 \quad$ Accessed 22 July 2021.

482 3. Moxon ER. Applications of molecular microbiology to vaccinology. Lancet Lond Engl 483 1997; 350:1240-1244.

484 4. Plotkin S. History of vaccination. Proc Natl Acad Sci 2014; 111:12283-12287. 
5. Overview of the implementation of COVID-19 vaccination strategies and vaccine deployment plans in the EU/EEA. 2021. Available at: https://www.ecdc.europa.eu/en/publications-data/overview-implementation-covid-19vaccination-strategies-and-vaccine-deployment. Accessed 2 June 2021.

6. Fournier P-E, Colson P, Levasseur A, et al. Emergence and outcomes of the SARS-CoV-2 'Marseille-4' variant. Int J Infect Dis 2021; 106:228-236.

7. Bedotto M, Fournier P-E, Houhamdi L, et al. Implementation of an in-house real-time reverse transcription-PCR assay for the rapid detection of the SARS-CoV-2 Marseille-4 variant. J Clin Virol 2021; 139:104814.

8. La Scola B, Le Bideau M, Andreani J, et al. Viral RNA load as determined by cell culture as a management tool for discharge of SARS-CoV-2 patients from infectious disease wards. Eur J Clin Microbiol Infect Dis 2020; 39:1059-1061.

9. Jones BE, Brown-Augsburger PL, Corbett KS, et al. The neutralizing antibody, LYCoV555, protects against SARS-CoV-2 infection in nonhuman primates. Sci Transl Med 2021; 13. Available at: https://stm.sciencemag.org/content/13/593/eabf1906. Accessed 5 July 2021.

10. Chi X, Yan R, Zhang J, et al. A neutralizing human antibody binds to the N-terminal domain of the Spike protein of SARS-CoV-2. Science 2020; 369:650-655.

11. Froimowitz M. HyperChem: a software package for computational chemistry and molecular modeling. BioTechniques 1993; 14:1010-1013.

12. Fantini J, Chahinian H, Yahi N. Synergistic antiviral effect of hydroxychloroquine and azithromycin in combination against SARS-CoV-2: What molecular dynamics studies of virus-host interactions reveal. Int J Antimicrob Agents 2020; 56:106020.

13. Fantini J, Yahi N, Azzaz F, Chahinian H. Structural dynamics of SARS-CoV-2 variants: A health monitoring strategy for anticipating Covid-19 outbreaks. J Infect 2021; Available at: https://www.ncbi.nlm.nih.gov/pmc/articles/PMC8172274/. Accessed 5 July 2021. 
511 14. Kruglova N, Siniavin A, Gushchin V, Mazurov D. Different Neutralization Sensitivity of 512 SARS-CoV-2 Cell-to-Cell and Cell-Free Modes of Infection to Convalescent Sera. Viruses $513 \quad 2021 ; 13: 1133$.

15. Chen X, Pan Z, Yue S, et al. Disease severity dictates SARS-CoV-2-specific neutralizing antibody responses in COVID-19. Signal Transduct Target Ther 2020; 5:180.

16. Legros V, Denolly S, Vogrig M, et al. A longitudinal study of SARS-CoV-2-infected patients reveals a high correlation between neutralizing antibodies and COVID-19 severity. Cell Mol Immunol 2021; 18:318-327.

17. Zucman N, Uhel F, Descamps D, Roux D, Ricard J-D. Severe Reinfection With South African Severe Acute Respiratory Syndrome Coronavirus 2 (SARS-CoV-2) Variant 501Y.V2. Clin Infect Dis 2021; :ciab129.

18. Network for Genomic Surveillance in South Africa, COMMIT-KZN Team, Cele S, et al. Escape of SARS-CoV-2 501Y.V2 from neutralization by convalescent plasma. Nature 2021; 593:142-146.

19. Avanzato VA, Matson MJ, Seifert SN, et al. Case Study: Prolonged Infectious SARS-CoV2 Shedding from an Asymptomatic Immunocompromised Individual with Cancer. Cell 2020; 183:1901-1912.e9.

20. Choi B, Choudhary MC, Regan J, et al. Persistence and Evolution of SARS-CoV-2 in an Immunocompromised Host. N Engl J Med 2020; 383:2291-2293.

21. Greaney AJ, Loes AN, Crawford KHD, et al. Comprehensive mapping of mutations in the SARS-CoV-2 receptor-binding domain that affect recognition by polyclonal human plasma antibodies. Cell Host Microbe 2021; 29:463-476.e6.

22. Andreano E, Piccini G, Licastro D, et al. SARS-CoV-2 escape in vitro from a highly neutralizing COVID-19 convalescent plasma. Immunology, 2020. Available at: http://biorxiv.org/lookup/doi/10.1101/2020.12.28.424451. Accessed 31 May 2021. 
23. Li Q, Wu J, Nie J, et al. The Impact of Mutations in SARS-CoV-2 Spike on Viral Infectivity and Antigenicity. Cell 2020; 182:1284-1294.e9.

24. Wang Z, Schmidt F, Weisblum $\mathrm{Y}$, et al. mRNA vaccine-elicited antibodies to SARS-CoV-2 and circulating variants. bioRxiv 2021; :2021.01.15.426911.

25. Weisblum Y, Schmidt F, Zhang F, et al. Escape from neutralizing antibodies by SARSCoV-2 spike protein variants. eLife 2020; 9:e61312.

26. Mlcochova P, Kemp S, Dhar MS, et al. SARS-CoV-2 B.1.617.2 Delta variant emergence and vaccine breakthrough. bioRxiv 2021; :2021.05.08.443253.

27. Planas D, Veyer D, Baidaliuk A, et al. Reduced sensitivity of infectious SARS-CoV-2 variant B.1.617.2 to monoclonal antibodies and sera from convalescent and vaccinated individuals. Microbiology, 2021. Available at: http://biorxiv.org/lookup/doi/10.1101/2021.05.26.445838. Accessed 2 June 2021.

28. Commissioner O of the. Coronavirus (COVID-19) Update: FDA Revokes Emergency Use Authorization for Monoclonal Antibody Bamlanivimab. FDA, 2021. Available at: https://www.fda.gov/news-events/press-announcements/coronavirus-covid-19-update-fdarevokes-emergency-use-authorization-monoclonal-antibody-bamlanivimab. Accessed 23 June 2021.

29. An EUA for Bamlanivimab-A Monoclonal Antibody for COVID-19. JAMA 2021; 325:880.

30. Taylor PC, Adams AC, Hufford MM, de la Torre I, Winthrop K, Gottlieb RL. Neutralizing monoclonal antibodies for treatment of COVID-19. Nat Rev Immunol 2021; 21:382-393.

31. Garcia-Beltran WF, Lam EC, St. Denis K, et al. Multiple SARS-CoV-2 variants escape neutralization by vaccine-induced humoral immunity. Cell 2021; 184:2523.

32. Hoffmann M, Arora P, Groß R, et al. SARS-CoV-2 variants B.1.351 and P.1 escape from neutralizing antibodies. Cell 2021; 184:2384-2393.e12. 
33. Piccoli L, Park Y-J, Tortorici MA, et al. Mapping Neutralizing and Immunodominant Sites on the SARS-CoV-2 Spike Receptor-Binding Domain by Structure-Guided HighResolution Serology. Cell 2020; 183:1024-1042.e21.

34. Voss WN, Hou YJ, Johnson NV, et al. Prevalent, protective, and convergent IgG recognition of SARS-CoV-2 non-RBD spike epitopes. Science 2021; 372:1108-1112.

35. Wibmer CK, Ayres F, Hermanus T, et al. SARS-CoV-2 501Y.V2 escapes neutralization by South African COVID-19 donor plasma. Nat Med 2021; 27:622-625.

36. Polack FP, Thomas SJ, Kitchin N, et al. Safety and Efficacy of the BNT162b2 mRNA Covid-19 Vaccine. N Engl J Med 2020; 383:2603-2615.

37. Point épidémiologique COVID-19 du 17 juin 2021 : nette diminution de la circulation du SARS-CoV-2, les mesures de prévention et la vaccination restent indispensables. Available at: https://www.santepubliquefrance.fr/presse/2021/point-epidemiologique-covid-19-du-17juin-2021-nette-diminution-de-la-circulation-du-sars-cov-2-les-mesures-de-prevention-etla-vaccination-rest. Accessed 23 June 2021.

38. Fournier P-E, Raoult D. Evaluating vaccination against SARS-CoV-2. The Lancet Available at: https://www.mediterranee-infection.com/wpcontent/uploads/2020/04/THELANCET-S-21-05441-article-soumis-le-28.05.2021.pdf.

39. Hasan A, Al-Ozairi E, Al-Baqsumi Z, Ahmad R, Al-Mulla F. Cellular and Humoral Immune Responses in Covid-19 and Immunotherapeutic Approaches. ImmunoTargets Ther 2021; 10:63-85.

40. Melgaço JG, Brito e Cunha D, Azamor T, et al. Cellular and Molecular Immunology Approaches for the Development of Immunotherapies against the New Coronavirus (SARS-CoV-2): Challenges to Near-Future Breakthroughs. J Immunol Res 2020; 2020:121.

41. Grifoni A, Weiskopf D, Ramirez SI, et al. Targets of T Cell Responses to SARS-CoV-2 Coronavirus in Humans with COVID-19 Disease and Unexposed Individuals. Cell 2020; 181:1489-1501.e15. 
588 42. Coste AT, Jaton K, Papadimitriou-Olivgeris M, Greub G, Croxatto A. Comparison of $589 \quad$ SARS-CoV-2 serological tests with different antigen targets. J Clin Virol 2021;

$590 \quad 134: 104690$.

591 43. Edouard S, Jaafar R, Orain N, et al. Automated Western immunoblotting detection of anti592 SARS-CoV-2 serum antibodies. Eur J Clin Microbiol Infect Dis 2021; 40:1309-1317.

$593 \quad$ Figure and Tables

594 Figure 1. Neutralizing response against the original virus (Original/B) and the 9 SARS595 CoV-2 variants in convalescent patients and in vaccinated participants. 
Figure 1

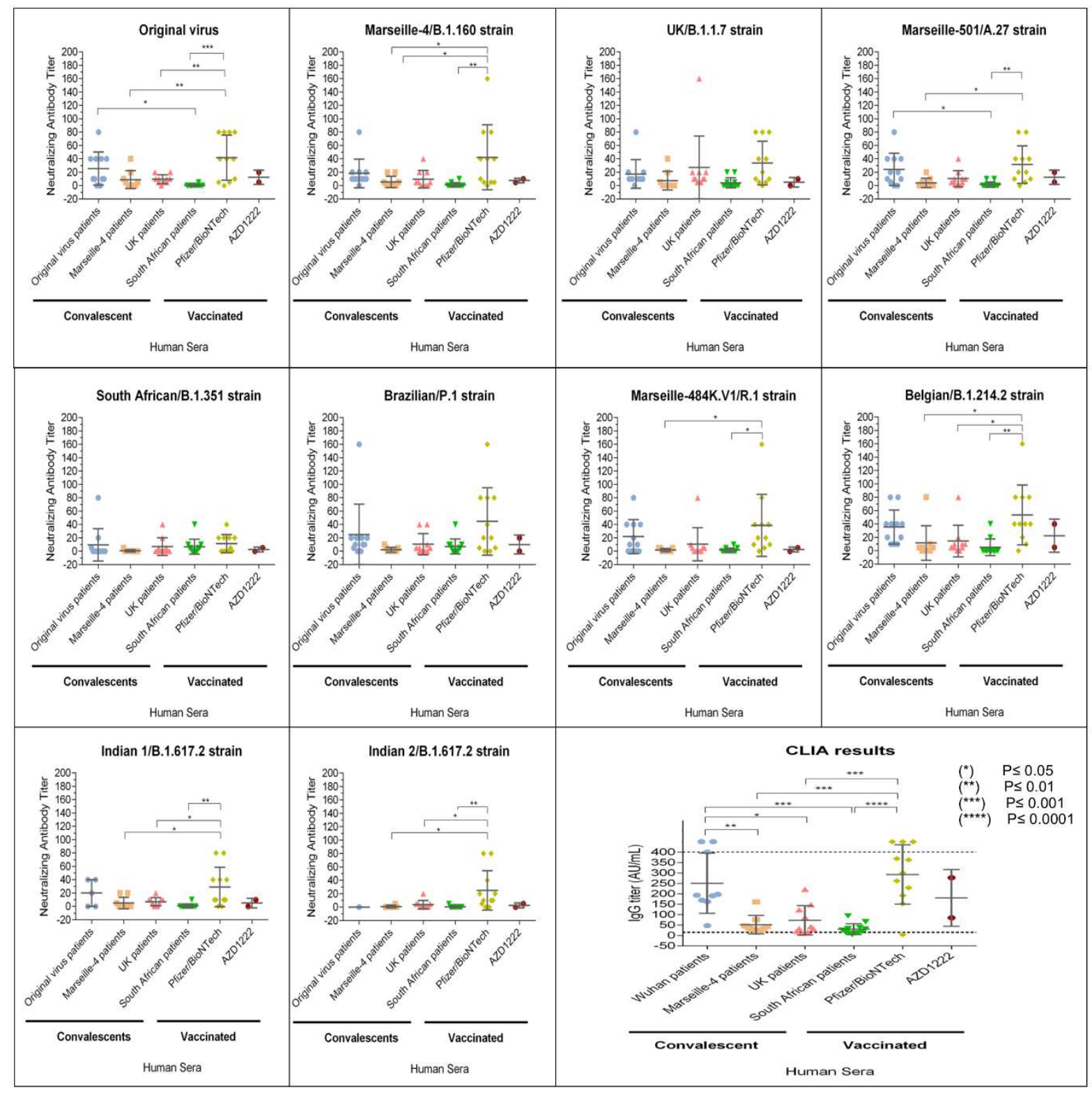

597 The Y-axis represents the neutralizing antibody titres obtained by MNT, and the X-axis represents the corresponding group of tested human sera. Data are shown as the mean and 
599 standard error of the mean (SEM). Solid lines represent the geometric mean titre, and the 600 whiskers show the $95 \%$ confidence interval. Each scatter represents one serum. Scatter symbols 601 and colours are attributed based on the sera group. Serology results are shown for the 602 convalescent patients and for the vaccinated patients. For seroneutralization, the 10 quadrants 603 correspond to one different tested strain each. The last quadrant shows the IgG titres in AU/mL 604 as obtained by chemiluminescent immunoassay (CLIA) for each serum group. IgG titres $>400$ 605 are represented in the graph above the maximum threshold of detection (400 AU/mL). The same 606 statistical significance was obtained by excluding these nonquantitative values $(>400)$ from the 607 ANOVA test. In all graphs, significance is represented by an asterisk for $\mathrm{P} \leq 0.05$. The absence of 608 an asterisk means no significant variance was detected between the groups. 

and the 9 SARS-CoV-2 variants in convalescent patients and in vaccinated participants.

Figure 2

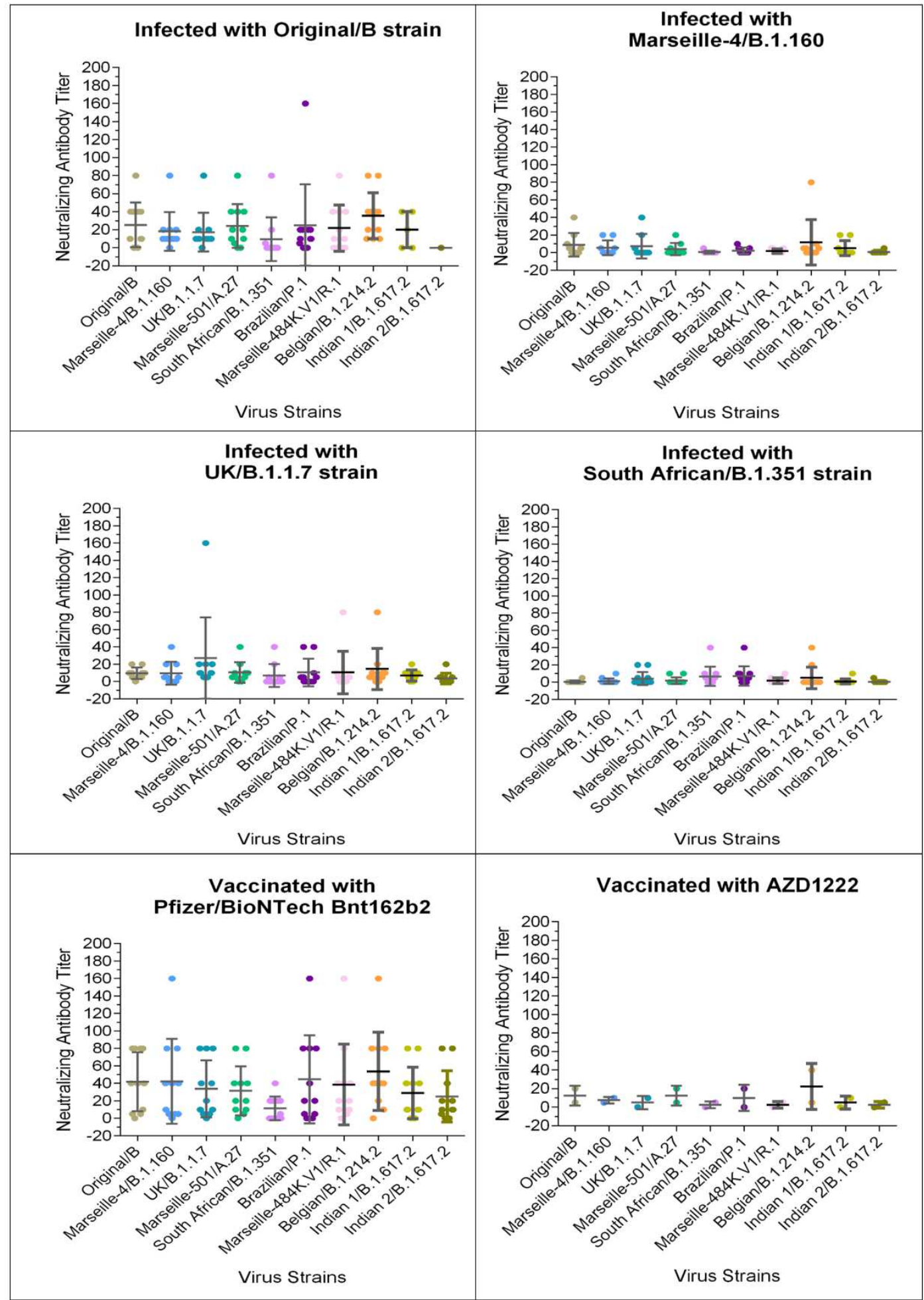


613 The $\mathrm{Y}$ axis represents the neutralizing antibody titres obtained by MNT, and the $\mathrm{X}$ axis consists 614 of the different tested SARS CoV2 strains. Data are shown as the mean and standard error of the 615 mean (SEM). Solid lines represent the geometric mean titre, and the whiskers show the 95\% 616 confidence interval. Each scatter represents one serum. Scatter's symbols and colours are strain 617 specific. Each quadrant in the figure corresponds to one different sera group. In all graphs, 618 significance is represented by an asterisk for $\mathrm{P} \leq 0.05$. Significance is represented as an asterisk 619 for significant p-values. The absence of an asterisk means no significant variance was detected 620 between groups.

621 
Figure 3. Variability of the main neutralizing epitope in the NTD among virus strains.

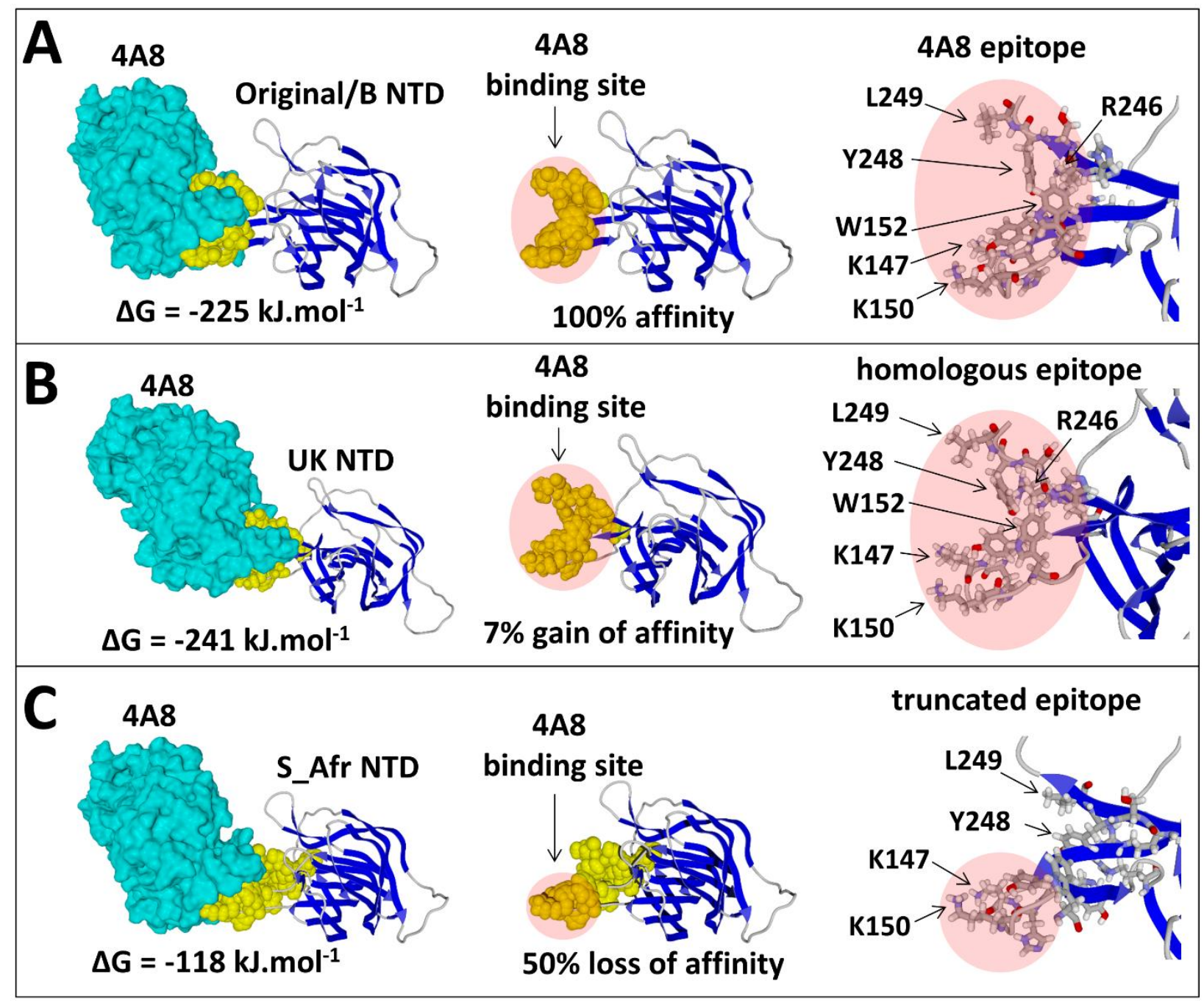

623

624

625

626

627

628

629

630

631

632

633

634

A. Molecular mechanism of NTD recognition (Original/B strain) by the 4A8 nAb. The NTDnAb complex (pdb file \#7C2 L) has a global affinity of $-225 \mathrm{~kJ} / \mathrm{mol}^{-1}$. The antibody clamps two distal zones of the NTD (the N3 loop with amino acid residues K147, K150 and W152) and the N5 loop (R246, Y248 and L249), which together form the main neutralizing epitope of the NTD. B. The NTD of the UK/B.1.1.7 variant retains this crescent-shaped structure, which displays a slightly higher affinity (+ 7\% compared with the Original/B strain) for the $4 \mathrm{~A} 8 \mathrm{nAb}$ due to the repositioning of the amino acids of the N3 loop. C. In the case of the S_Afr variant, only the N3 loop part of the epitope is conserved, so that the affinity for the $4 \mathrm{~A} 8 \mathrm{nAb}$ is decreased by $50 \%$. Such a truncated epitope may elicit a poor antibody response, consistent with seroneutralization data. 
Table 1. Immuno-escape index (I-index).

\begin{tabular}{|c|c|c|c|c|}
\hline Virus & $\begin{array}{c}\Delta \text { G RBD } \\
(\text { LyCoV-555) } \\
\text { kJ.mol }{ }^{-1}\end{array}$ & $\begin{array}{c}\Delta G \text { NTD } \\
(4 \mathrm{A8}) \\
\text { kJ.mol }\end{array}$ & 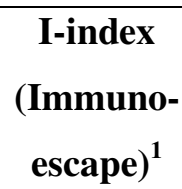 & $\begin{array}{c}\text { MNT } \geq 1 / 5 \\
(\mathrm{nb} / 55)\end{array}$ \\
\hline Original/B & -244 & -225 & 1.0 & $36 / 55(65 \%)$ \\
\hline Marseille-4 & -245 & -225 & 1.0 & $35 / 55(64 \%)$ \\
\hline Belgian & -210 & -225 & 1.1 & $39 / 55(71 \%)$ \\
\hline UK & -195 & 241 & 1.2 & $38 / 55(69 \%)$ \\
\hline South African & -75 & -118 & 2.6 & $21 / 55(38 \%)$ \\
\hline Brazilian & -80 & -82 & 2.9 & $34 / 55(62 \%)$ \\
\hline $\begin{array}{l}\text { Marseille- } \\
484 \mathrm{~K} . \mathrm{V} 1\end{array}$ & -36 & -258 & 3.0 & $28 / 55(51 \%)$ \\
\hline Mrs-501 & -59 & -114 & 3.1 & $34 / 55(62 \%)$ \\
\hline Indian 1 & -68 & -88 & 3.1 & $25 / 49(51 \%)$ \\
\hline Indian 2 & -68 & -76 & 3.3 & $16 / 41(39 \%)$ \\
\hline
\end{tabular}

637 Evaluation of the level of resistance of a SARS-CoV-2 variant to neutralizing antibodies (nAb)

638 directed against the RBD and the NTD of the spike protein. I-index is considered significant if $639>2 .(\mathrm{wt}=$ Original $/ \mathrm{B}$, mut=other $)$

$640 \quad \mathrm{I}$-index $=1 / 2\left(\Delta \mathrm{G}_{\mathrm{wt}} / \Delta \mathrm{G}_{\mathrm{mut}}[\mathrm{RBD}-\mathrm{nAb}]+\Delta \mathrm{G}_{\mathrm{wt}} / \Delta \mathrm{G}_{\mathrm{mut}}[\mathrm{NTD}-\mathrm{nAb}]\right.$

641 The formula was designed so that the reference virus retrieved from PDB files 7KMG and 7C2 L

642 had an I-index $=1$. Under these conditions, the variants of the present studies could be classified 643 into two groups: those with an I-index close to 1 (Marseille-4, Belgian and UK variants) that are 644 predicted to be efficiently neutralized by natural and/or vaccinal nAbs and those with an I-index 645 >2 (South Africa, Marseille-484K. V1, Marseille-501, Brazilian, Indian_1 and Indian_2) that are 646 likely to resist seroneutralization. 


\section{Supporting Informations}

649 Supporting table S1. Serology of convalescent patients.

650 Tables of data and serological testing results relative to all convalescent patients in the study; patients were identified as their 651 reference ID featuring also in Supporting Tables S4. The table includes the Sera ID, the sex of each participant (F/M), their birth year 652 and age, their CLIA IgG test results (AU/mL), and the time of plasma sample collection relative to days after the $1^{\text {st }}$ positive PCR 653 results for each patient. NA in CLIA results means no available serological test for the corresponding sera. 


\begin{tabular}{|c|c|c|}
\hline Sera ID & $\operatorname{Sex}(\mathbf{F} / \mathbf{M})$ & Year of birth \\
\hline I-1 & F & 1969 \\
\hline I-2 & $\mathrm{F}$ & 1971 \\
\hline I- 3 & M & 1960 \\
\hline I-4 & M & 1980 \\
\hline I-5 & M & 1982 \\
\hline I- 6 & F & 1986 \\
\hline I-7 & M & 1943 \\
\hline I-8 & M & 1968 \\
\hline I-9 & F & 1965 \\
\hline I-10 & M & 1964 \\
\hline I-11 & M & 2006 \\
\hline II-1 & $\mathrm{F}$ & 1968 \\
\hline II-2 & F & 1995 \\
\hline II-3 & F & 1993 \\
\hline II-4 & F & 1975 \\
\hline II- 5 & M & 1956 \\
\hline II-6 & M & 1935 \\
\hline II-7 & M & 1981 \\
\hline II-8 & M & 1952 \\
\hline II-9 & $\mathrm{F}$ & 2004 \\
\hline UK-1 & M & 1986 \\
\hline UK-2 & M & 1962 \\
\hline UK-3 & M & 1965 \\
\hline UK-4 & $\mathrm{F}$ & 1994 \\
\hline UK-5 & M & 1960 \\
\hline UK-6 & F & 1995 \\
\hline UK-7 & M & 1956 \\
\hline UK-8 & F & 1993 \\
\hline UK-9 & M & 1992 \\
\hline UK-10 & F & 1981 \\
\hline SA-1 & F & 1994 \\
\hline $\mathrm{SA}-2$ & M & 1985 \\
\hline $\mathrm{SA}-3$ & F & 1997 \\
\hline $\mathrm{SA}-4$ & F & 1980 \\
\hline SA-5 & M & 1968 \\
\hline SA-6 & $\mathrm{F}$ & 1974 \\
\hline SA-7 & M & 1974 \\
\hline SA-8 & $\mathrm{F}$ & 1992 \\
\hline SA-9 & F & 1963 \\
\hline SA-10 & F & 1978 \\
\hline SA-11 & F & 1971 \\
\hline SA-12 & $\mathrm{F}$ & 1986 \\
\hline
\end{tabular}


657 Tables of data and serological testing results relative to vaccinated participants in the study; they were identified as their reference ID 658 featuring Supporting Table S5. The table includes the Sera ID, the sex of each participant (F/M), their birth year and age, their CLIA $659 \mathrm{IgG}$ test results $(\mathrm{AU} / \mathrm{mL})$, and the time of plasma sample collection relative to the period after their second-shot vaccine uptake.

\begin{tabular}{cccc}
\hline Sera ID & Sex (F/M) & Year of birth & Age (years) \\
\hline V-Pfizer-1 & M & 1967 & 54 \\
V-Pfizer-2 & M & 1961 & 60 \\
V-Pfizer-3 & F & 1969 & 52 \\
\hline V-Pfizer-4 & M & 1952 & 69 \\
V-Pfizer-5 & F & 1969 & 52 \\
\hline V-Pfizer-6 & F & 1994 & 27 \\
V-Pfizer-7 & M & 1992 & 29 \\
V-Pfizer-8 & F & 1993 & 28 \\
V-Pfizer-9 & M & 1992 & 29 \\
V-Pfizer-10 & M & 1939 & 82 \\
V-Pfizer-11 & M & 1992 & 29 \\
Astra-1 & F & 1963 & 58 \\
Astra-2 & M & 1963 & 58 \\
\hline
\end{tabular}

660 
663 Table extending related genomic information for each of the tested SARS CoV2 strains in our study. Clade, lineage, name of IHU

664 isolate and the corresponding spike amino acid mutations (substitutions and deletions) are shown for each strain. NI= Not Identified.

\begin{tabular}{|c|c|c|c|c|c|}
\hline Strain name & $\begin{array}{l}\text { Clade } \\
\text { (Next Clade) }\end{array}$ & $\begin{array}{l}\text { PANGO } \\
\text { lineage }\end{array}$ & $\mathrm{IHU}$ isolate & Spike amino acid substitutions & Spike amino acid deletions \\
\hline Original/B virus & $20 \mathrm{~B}$ & $\mathrm{~B}$ & IHUMI717 & D614G, R682P & $\mathrm{NI}$ \\
\hline Marseille-4 variant & $20 \mathrm{~A}$ & B.1.160 & IHUMI2096 & S477N, D614G & $\mathrm{NI}$ \\
\hline Marseille-501 variant & $19 \mathrm{~B}$ & A. 27 & IHUMI3217 & L18F, L452R, N501Y, A653V, H655Y, D796Y, D843N, G1219V & $\mathrm{NI}$ \\
\hline $\begin{array}{l}\text { Marseille-484K.V1 } \\
\text { variant }\end{array}$ & $20 \mathrm{~B}$ & R.1 & IHUMI3239 & W152L, E484K, D614G, G769V & $\mathrm{NI}$ \\
\hline Belgian variant & $20 \mathrm{~A}$ & B.1.214.2 & IHUMI3246 & Q414K, G446D, N450K, D614G, T716I, Y837D & $\mathrm{NI}$ \\
\hline UK variant & $\begin{array}{l}201 \\
\text { (Alpha, V1) }\end{array}$ & B.1.1.7 & IHUMI3076 & N501Y, A570D, D614G, P681H, T716I, S982A, D1118H & H69-, V70-, Y144- \\
\hline South African variant & $\begin{array}{l}20 \mathrm{H} \\
\text { (Beta, V2) }\end{array}$ & B.1.351 & IHUMI3147 & L18F, D80A, D215G, L242H, K417N, E484K, N501Y, D614G, A701V & A243-, L244-, H245- \\
\hline Brazilian variant & $\begin{array}{l}20 \mathrm{~J} \\
\text { (Gamma, V3) }\end{array}$ & $\begin{array}{l}\text { B.1.1.28.1 } \\
\text { (P1) }\end{array}$ & IHUMI3191 & $\begin{array}{l}\text { L18F, T20N, P26S, D138Y, R190S, K417T, E484K, N501Y, D614G, H655Y, R682W, T1027I, } \\
\text { V1176F }\end{array}$ & $\mathrm{NI}$ \\
\hline Indian 1 variant & 21A (Delta) & B.1.617.2 & IHUMI3396 & T19R, A67V, T95I, R158G, L452R, T478K, D614G, P681R, R682Q & E156-, F157- \\
\hline Indian 2 variant & $21 \mathrm{~A}$ (Delta) & B.1.617.2 & IHUMI3630 & T19R, R158G, A222V, L452R, T478K, D614G, P681R, R682W, D950N, S1252F & E156-, F157- \\
\hline
\end{tabular}


667 Supporting table S4. Seroneutralization results for convalescent patients.

668 Table extending the neutralization results of the 42 serum samples from the convalescent patient participating in our study as tested 669 against 10 different strains of SARS-CoV-2. Monoclonal antibodies against bamlanivimab (LY-CoV 555) were used as controls. IgG 670 titres tested by CLIA are presented in AU/mL. Human sera are divided into four different categories (Wuhan/B, Marseille-4/B.1.160,

671 United Kingdom, South African/B.1.351) related to previous infection. Seroneutralisation titres legend are shown on the right. Titres 672 for human sera are represented in the red gradient, and titres for mAbs are represented in the grey gradient. NA= Not Available 673 results. The absence of neutralization results is marked as white cases. 


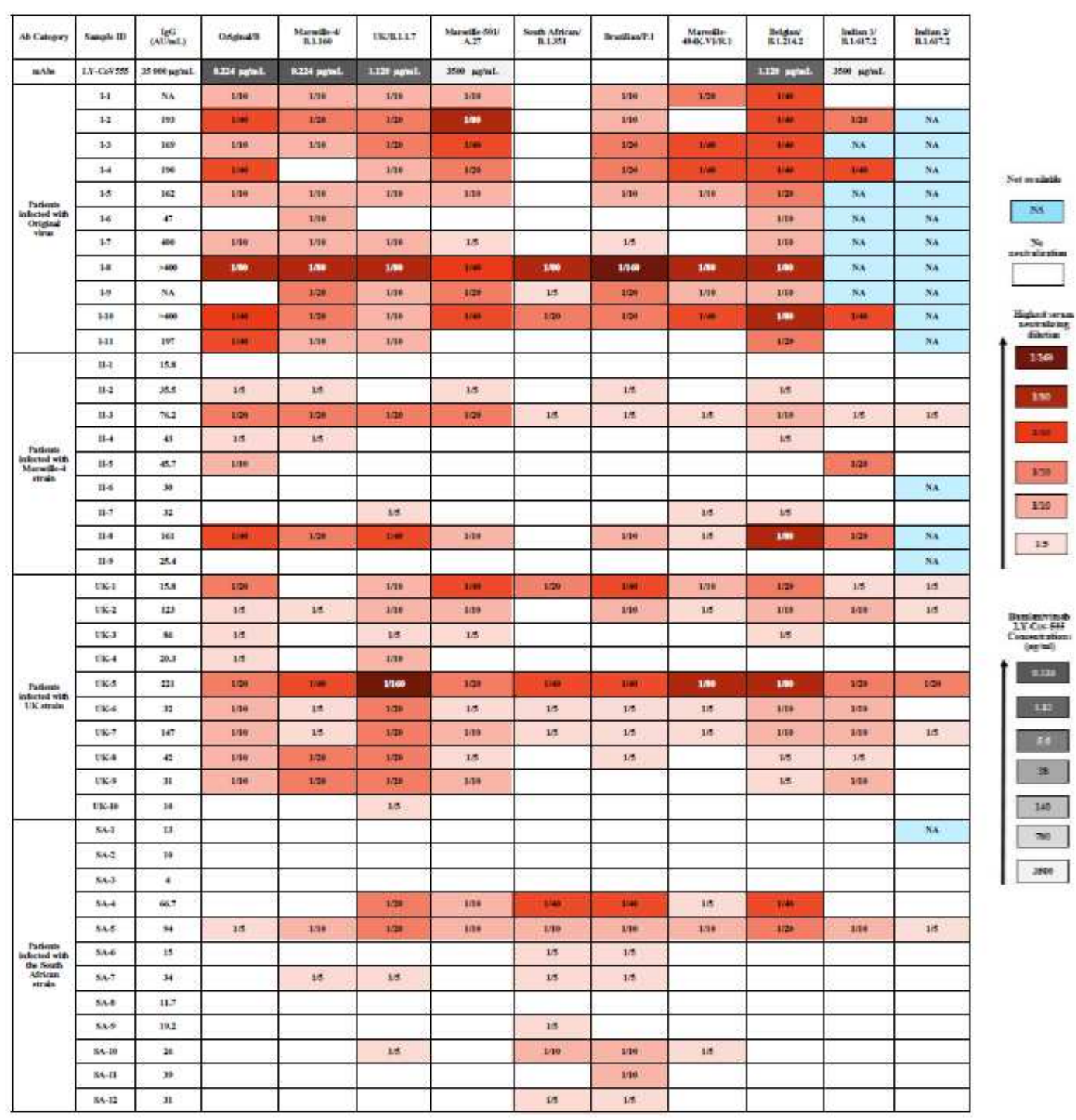




\section{Supporting table S5. Seroneutralisation results for vaccinated participants.}

677 Table extending the neutralization results of the 13 serum samples from the vaccinated participants in our study against 10 different 678 strains of SARS-CoV-2. Monoclonal antibodies against bamlanivimab (LY-CoV 555) were used as controls. IgG titres tested by 679 CLIA are presented in AU/mL. Human sera are divided into two different categories (mRNA-based Pfizer/BioNTech vaccine and 680 adenovirus-based AZD1222 vaccine) based on vaccine type. Seroneutralization titre legends are shown on the right of each table 681 (titres for human sera are represented in the red gradient, and titres for mAbs are represented in the grey gradient). The absence of 682 neutralization results is marked as white cases.

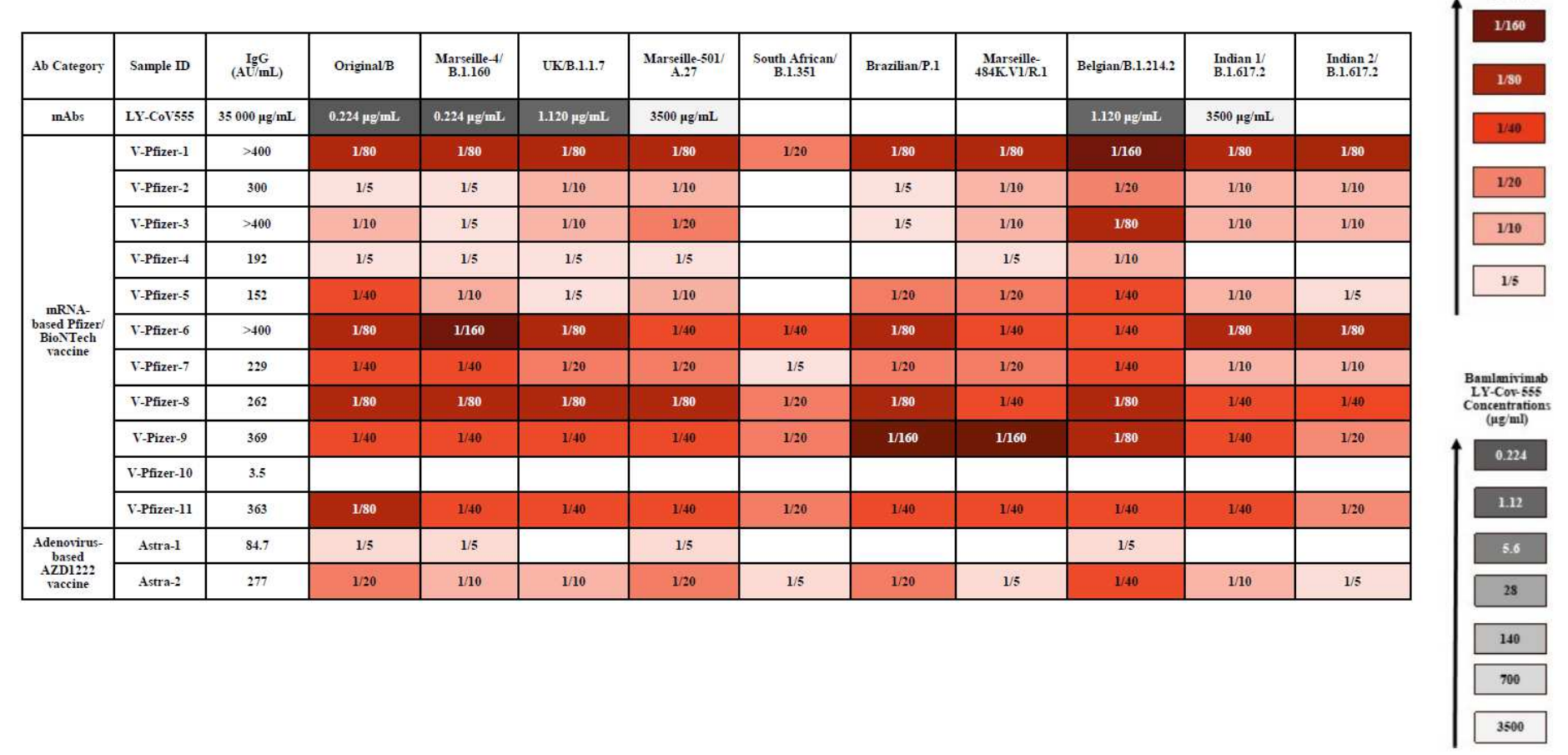


685 Supporting Figure S1. Neutralizing activity of the monoclonal antibody bamlanivimab.

686 Table showing the neutralizing activity of LY-CoV555 against the 10 tested strains. Neutralizing concentrations are represented by 687 grey colour gradient cases and are displayed in $\mu \mathrm{g} / \mathrm{mL}$ units. Darker grey colours reflect higher neutralization activity.

\section{LY-COV555}

\begin{tabular}{|l|l|}
\hline Virus strains & $\begin{array}{l}\text { Neutrilazing mAbs } \\
\text { concentration }\end{array}$ \\
\hline South African/B.1.351 & \\
\hline Brazillian/P.1 & \\
\hline Marseille-484K.V1/ R.1 & \\
\hline Indian 2/B.1.617.2 & \\
\hline Indian 1/B.1.617.2 & $3500 \mu \mathrm{g} / \mathrm{mL}$ \\
\hline Marseille-501/A.27 & $3500 \mu \mathrm{g} / \mathrm{mL}$ \\
\hline UK/B.1.1.7 & $1.120 \mu \mathrm{g} / \mathrm{mL}$ \\
\hline Belgian/B.1.214.2 & $1.120 \mu \mathrm{g} / \mathrm{mL}$ \\
\hline Original/B & $0.224 \mu \mathrm{g} / \mathrm{mL}$ \\
\hline Marseille-4/B.1.160 & $0.224 \mu \mathrm{g} / \mathrm{mL}$ \\
\hline
\end{tabular}

Absence of

neutralizing activity 
690 Supporting Figure S2. Molecular mechanisms of nAb escape in the RBD of SARS-CoV-2 variants.

691 A. The epitope recognized by the LY-CoV555 nAb (pdb file \#7KMG) consists of several amino acid (coloured in green) residues 692 distributed on the surface of the RBD. The anionic carboxylic group of E484 interacts with the cationic charge of R50 (heavy chain of 693 LY-CoV555 nAb) through an electrostatic bridge. The aromatic ring of Y490 interacts with a methyl group of I52 (heavy chain of 694 LY-CoV555 nAb) by a CH- $\pi$ interaction, which is reinforced by vicinal apolar amino acid residues (I54 and I55). B. In the Marseille695 484K.V1 variant, E484 (in red in the left panel) is mutated in E484K (in blue in the right panel). The consequence of this mutation is a 696 shift of the side chain of E484K whose cationic group (which replaces the negative charge of E484) now forms a cation- $\pi$ bond with 697 the aromatic ring of F490. In this new context, neither E484K nor F490 can still interact with the LY-CoV555 nAb. Indeed, R50, I52, 698 I54 and L55 of the heavy chain of the antibody are no longer involved in RBD recognition. C. Mutational pattern of the Marseille699 501/A.27 variant (L452R/N501Y). D. Mutational pattern of the India_1 variant (L452R/T478K). The same molecular modelling 700 method was applied to all variants (B-D) after introducing the mutations in the reference Original/B-nAb complex (PDB file \#7KMG) 701 followed by energy minimization of the RBD and simulations of the binding reaction. 


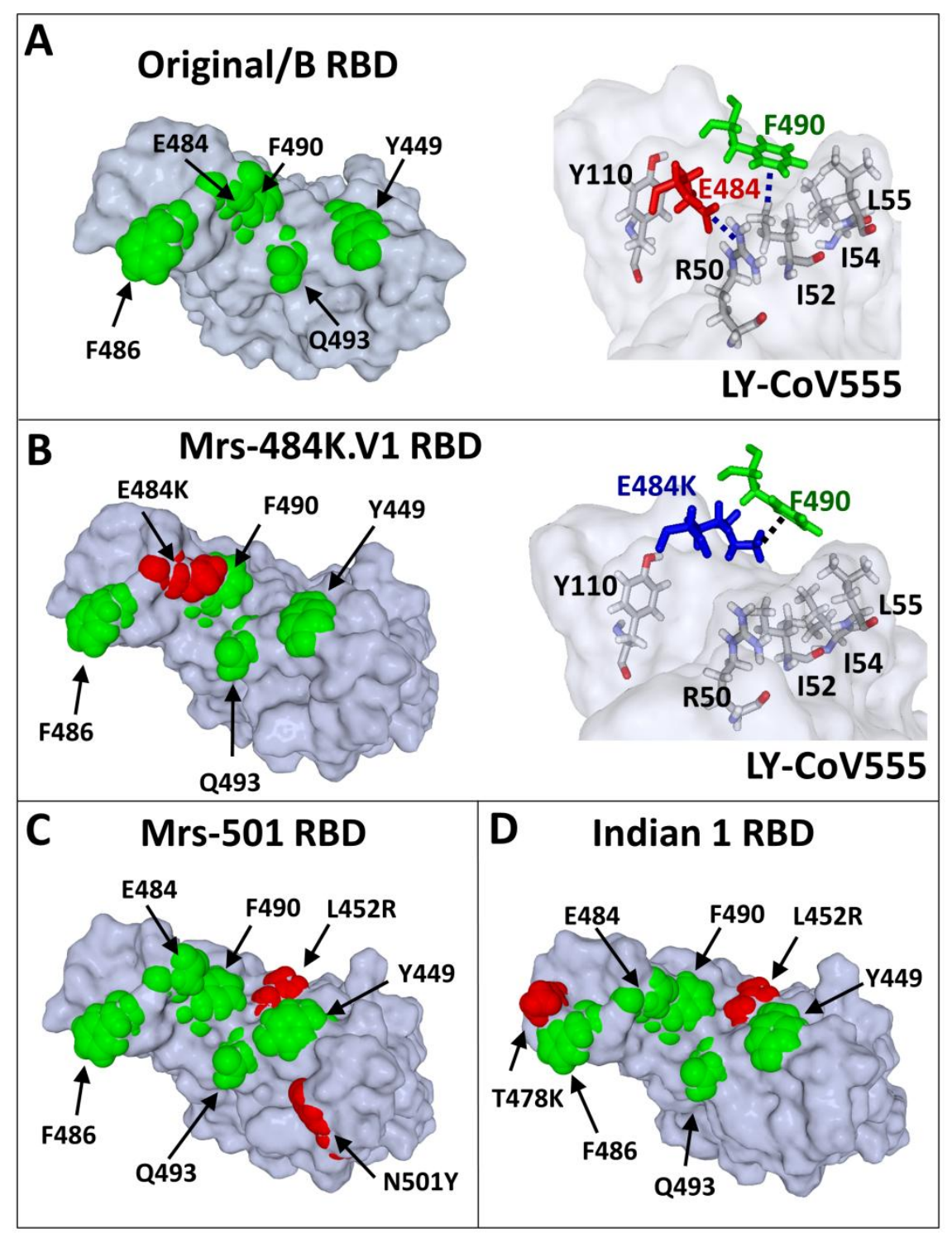


704 Supporting Figure S3. Molecular mechanism of nAb escape by the RBD of the Marseille-501 (Mrs-501) variant.

705 A. In the Original/B RBD (PDB file \#7KMG), the heavy chain of the LY-CoV 555 nAb interacts with the side chains of L452 (van 706 der Waals network) and Y449 (NH- $\pi$ ). B. In the Marseille-501/A.27 variant, the mutant R452 is displaced out of the van der Waals 707 network, which reorients Y449 so that the NH- $\pi$ interaction with N-31 is no longer possible. The aromatic ring of Y501 comes closer to Y449, which definitely prevents any contact with the heavy chain of the nAb. 


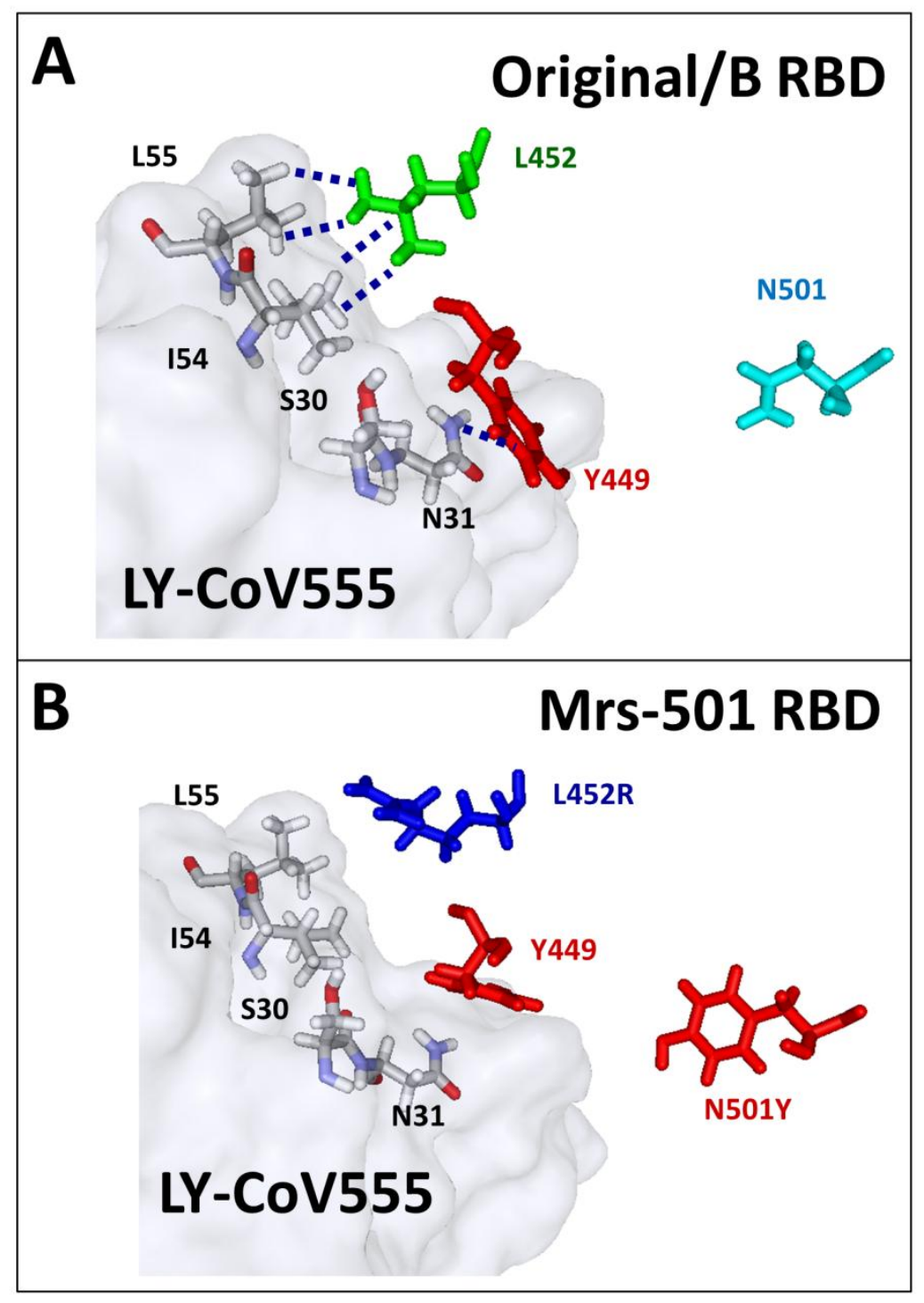


711 Supporting Figure S4. Molecular mechanism of nAb escape by the RBD of the Indian 1 variant.

712 A. A $\pi-\pi$ aromatic cluster is involved in the recognition of the RBD (Original/B strain) by the LY-CoV555 nAb (pdb file \#7KMG).

713 Y32 and Y92 of the light chain of the antibody clamp the aromatic ring of RBD residue F486. This cluster is stabilized by a CH- $\pi$

714 interaction between the methyl groups of T478 and F486. It should be noted that this interaction is important to functionally orient and

715 wedge the side chain of F486 between Y32 and Y92. B. In the Indian 1 variant, the mutation T478K prevents the formation of this

716 network by forming a cation- $\pi$ interaction between the cationic group of this residue and the aromatic ring of F486. This new bond

717 reorients the side chain of F486 towards the RBD surface, thereby preventing any possibility of association with the antibody. The

718 models are shown in sphere (upper panels) or stick (lower panels) representations. 


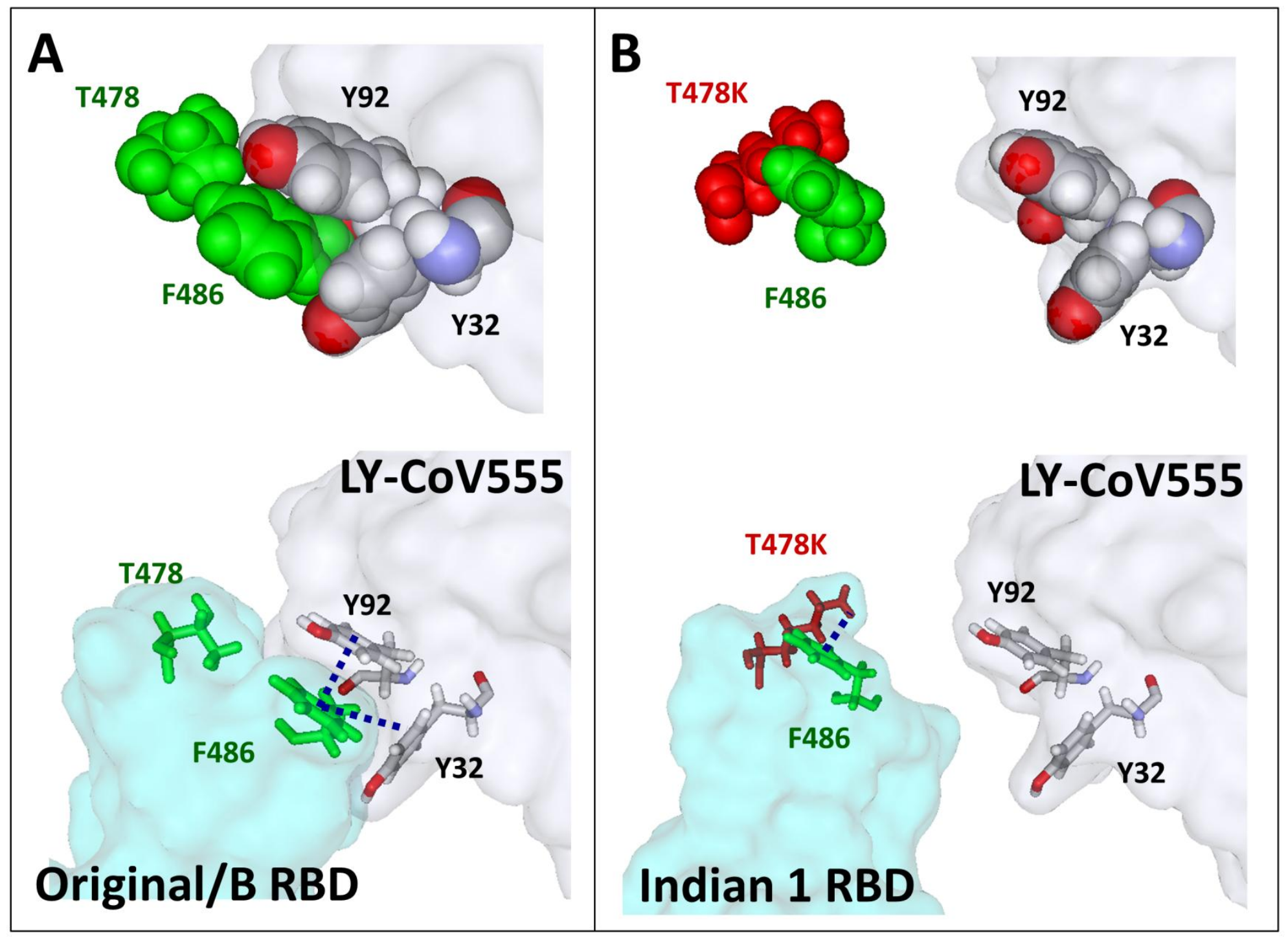


721 Supporting Figure S5. Intralineage variability of the main neutralizing epitope in the NTD: the case of India variants.

722 A. Indian 1 variant. B. Indian 2 variant. The left panels in A and B show the positions of residues K147 and R246 that face the 723 antibody. The superposition of the secondary structure shows that the conformational changes between these variants chiefly involve 724 the amino acid side chain orientation. In the case of Indian 1, both K147 and R246 interact with the antibody, whereas in the case of 725 Indian 2, R246 moves away the antibody, whereas K147 gets closer, resulting in decreased binding to avoid steric clash. The middle 726 panels show the NTD surface as "seen" by the antibody. The right panels show the subtle rearrangements of the NTD structure of both 727 India variants. The main change concerns the orientation of K147. 


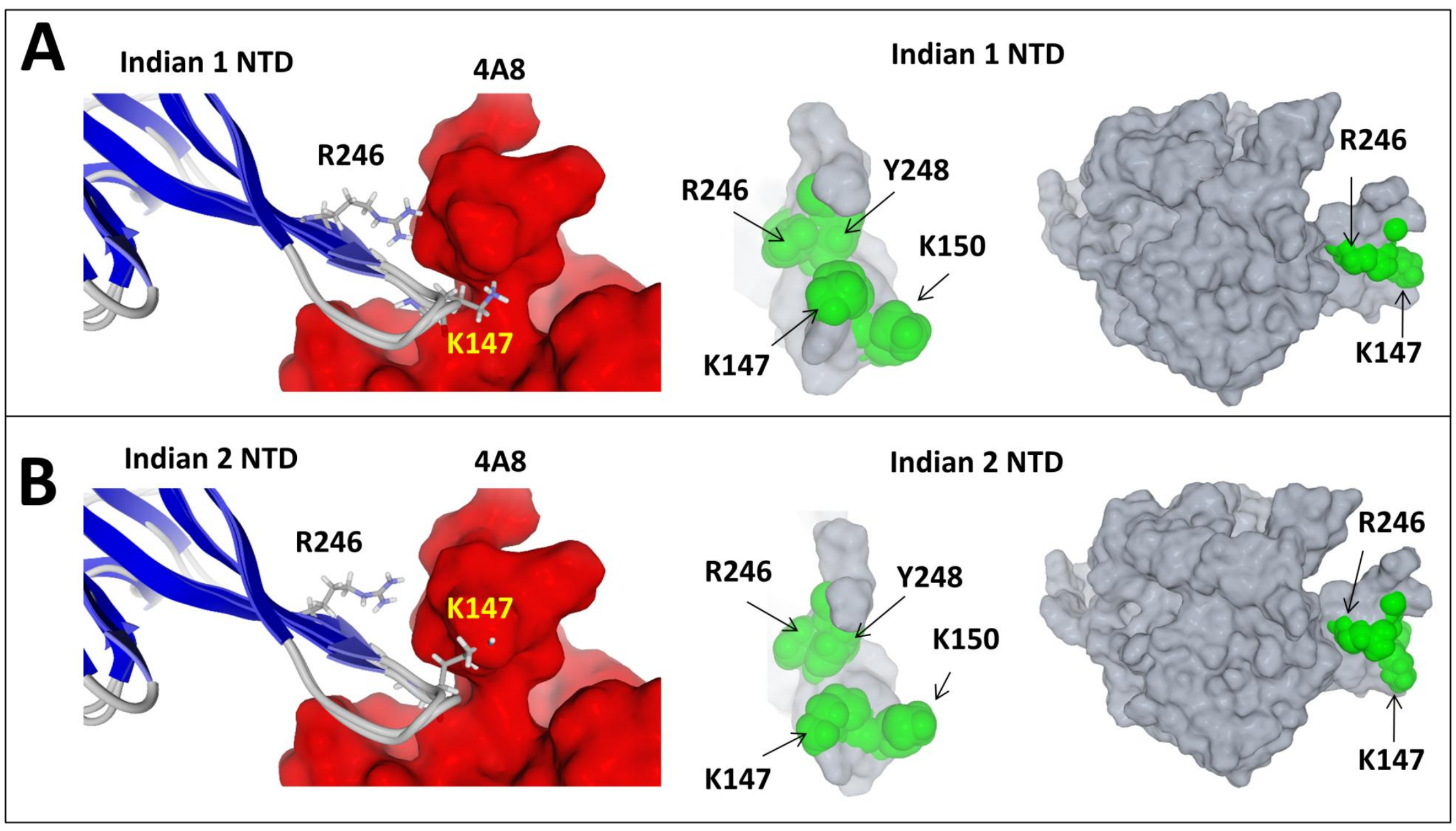

